\title{
MINIMAL IDENTITIES OF SYMMETRIC MATRICES
}

\author{
MA WENXIN AND MICHEL L. RACINE
}

Abstract. Let $H_{n}(F)$ denote the subspace of symmetric matrices of $M_{n}(F)$, the full matrix algebra with coefficients in a field $F$. The subspace $H_{n}(F) \subset$ $M_{n}(F)$ does not have any polynomial identity of degree less than $2 n$. Let

$$
T_{k}^{i}\left(x_{1}, \ldots, x_{k}\right)=\sum_{\substack{\sigma \in \mathscr{S}_{k} \\ 1 \leq i \leq k, \sigma^{-1}(i) \equiv 1,2 \bmod 4}}(-1)^{\sigma} x_{\sigma(1)} x_{\sigma(2)} \cdots x_{\sigma(k)},
$$

and $e(n)=n$ if $n$ is even, $n+1$ if $n$ is odd. For all $n \geq 1, T_{2 n}^{i}$ is an identity of $H_{n}(F)$. If the characteristic of $F$ does not divide $e(n)$ ! and if $n \neq 3$, then any homogeneous polynomial identity of $H_{n}(F)$ of degree $2 n$ is a consequence of $T_{2 n}^{i}$. The case $n=3$ is also dealt with. The proofs are algebraic, but an equivalent formulation of the first result in graph-theoretical terms is given.

\section{INTRODUCTION}

Razmyslov [5] introduced the concept of weak identities, namely, polynomials which evaluate to zero on some fixed subspace $W$ of an algebra $A$. We wish to determine the weak identities of minimal degree for the subspace $H_{n}(F)$ of symmetric matrices of the full matrix algebra $M_{n}(F)$. We refer to these as identities of $H_{n}$. Amitsur and Levitzki [1, Theorems 1 and 3] have shown that $M_{n}(F)$ has no polynomial identity of degree less than $2 n$ and that if $n>2$ or $|F|>2$ then any polynomial identity of $M_{n}$ of degree $2 n$ is a scalar multiple of the standard polynomial $S_{2 n}$, where

$$
S_{k}\left(x_{1}, x_{2}, \ldots, x_{k}\right)=\sum_{\sigma \in \mathscr{F}_{k}}(-1)^{\sigma} x_{\sigma(1)} x_{\sigma(2)} \cdots x_{\sigma(k)},
$$

$\mathscr{S}_{k}$ the symmetric group on $k$ objects and $(-1)^{\sigma}$ the sign of the permutation $\sigma$. In particular $S_{2 n}$ is an identity of $H_{n}$. An easy substitution argument [9, Proposition 2] shows there are no identities of $H_{n}$ of degree less than $2 n$.

Received by the editors September 15, 1988.

1980 Mathematics Subject Classification (1985 Revision). Primary 16A38; Secondary 05C50, $17 \mathrm{C} 05$.

The research of the second author was supported in part by an NSERC grant and by an SSHN fellowship of the French Foreign Ministry. 
To state our main result we must introduce other polynomials. Let

$$
T_{k}^{i}\left(x_{1}, \ldots, x_{k}\right)=\sum_{\substack{\sigma \in \mathscr{S}_{k} \\ 1 \leq i \leq k, \sigma^{-1}(i) \equiv 1,2 \bmod 4}}(-1)^{\sigma} x_{\sigma(1)} \cdots x_{\sigma(k)},
$$

Let $[a, b]$ denote the commutator $a b-b a$ and $\{a b c\}$ the triple product $a b c+c b a$. Let

$$
Q\left(x_{1}, x_{2}, x_{3}, x_{4}, x_{5}, x_{6}\right)=\sum_{\substack{(123) \\(456)}}\left\{\left[x_{1}, x_{2}\right]\left[x_{3}, x_{4}\right]\left[x_{5}, x_{6}\right]\right\},
$$

where the commutators are the arguments of the triple product and the sum is taken over cyclic permutations of 123 and 456 , so that $Q$ is the sum of nine triple products. One checks easily that $T_{k}$ and $Q$ are not the zero polynomial irrespective of the characteristic of the base field. We will prove

Theorem 1. Let $F$ be a field of arbitrary characteristic. For all $n \geq 1$ polynomials $T_{2 n}^{i}\left(x_{1}, x_{2}, \ldots, x_{2 n}\right)$ are identities of $H_{n}(F)$ and $Q\left(x_{1}, x_{2}, x_{3}, x_{4}, x_{5}, x_{6}\right)$ is an identity of $\mathrm{H}_{3}$.

Let $e(n)=2[(n+1) / 2]$.

Theorem 2. If characteristic $F \nmid e(n)$ ! and $|F|>2 n$, then for $n \neq 3$ all identities of $H_{n}(F)$ of degree $2 n$ are consequences of $T_{2 n}^{1}$. If $n=3$, then all identities of degree 6 of $H_{3}(F)$ are consequences of $T_{6}^{1}$ and $Q$.

\section{THE IDENTITIES}

In this section we prove Theorem 1.1. Razmyslov [6, p. 732] gave a proof of the Amitsur-Levitzki theorem, which shows that it follows from the fact that a matrix satisfies its characteristic polynomial. Rowen [8, Theorem 1] used a variation of this proof to prove Kostant's theorem that for even $n$ 's $S_{2 n-2}$ is an identity for skew-symmetric matrices. We in turn use a variation of Rowen's proof to prove Theorem 1.1 for $n$ even.

We start by recalling a few useful facts. Let $A$ be a central simple associative algebra over the field $F,^{*}$ an involution of $A$ of the first kind, i.e., $F \subset H\left(A,{ }^{*}\right)=\left\{a \in A \mid a^{*}=a\right\}$. The involution ${ }^{*}$ is said to be orthogonal if $\left(A \otimes \bar{F},{ }^{*}\right) \cong\left(M_{n}(\bar{F}), t\right)$, where $\bar{F}$ is the algebraic closure of $F$ and $t$ the transpose; it is said to be symplectic if $\left(A \otimes \bar{F},{ }^{*}\right) \cong\left(M_{n}(\bar{F}), s\right)$, $s$ the symplectic involution. In the last case $n$ must be even, say $n=2 m$. If ${ }^{*}$ is a symplectic involution of $A$, then the elements of $H\left(A,{ }^{*}\right)$ satisfy a polynomial of degree $m$ which is analogous to the characteristic polynomial but obtained using the Pfaffian instead of the determinant (for details see [3, p. 231]).

$$
x^{m}-\mu_{1} x^{m-1}+\mu_{2} x^{m-2}-\cdots+(-1)^{i} \mu_{i} x^{m-i}+\cdots+(-1)^{m} \mu_{m} .
$$

Just as in the case of the characteristic polynomial the coefficients $\mu_{i}$ are polynomials in traces of powers of $x$. If $\operatorname{char} F=0$ they are given inductively 
by

$$
\mu_{0}=1, \quad 2 k \mu_{k}=\sum_{i=1}^{k}(-1)^{i-1} \mu_{k-i} \operatorname{tr}\left(x^{i}\right) .
$$

These are the Newton identities with an extra 2, which comes in because the generic trace of $H\left(A,{ }^{*}\right),{ }^{*}$ symplectic, is half the reduced trace of $A$.

Recall

$$
\begin{aligned}
S_{k+1}\left(x_{1}, \ldots, x_{k+1}\right) & =\sum_{i=1}^{k+1}(-1)^{i-1} x_{i} S_{k}\left(x_{1}, \ldots, \hat{x}_{i}, \ldots, x_{k+1}\right) \\
& =\sum_{i=1}^{k+1}(-1)^{k+1-i} S_{k}\left(x_{1}, \ldots, \hat{x}_{i}, \ldots, x_{k+1}\right) x_{i} .
\end{aligned}
$$

If $n$ is even, two monomials which are cyclic permutations of one another have opposite signs in $S_{n}$. Since they have the same trace we have

$$
\operatorname{tr}\left(S_{2 k}\left(x_{1}, \ldots, x_{2 k}\right)\right)=0 \text {. }
$$

Similarly,

$$
\sum_{\sigma \in \mathscr{S}_{2 k+1}}(-1)^{\sigma} \operatorname{tr}\left(\left[y, x_{\sigma(1)}\right]\left[x_{\sigma(2)}, x_{\sigma(3)}\right] \cdots\left[x_{\sigma(2 k)}, x_{\sigma(2 k+1)}\right]\right)=0,
$$

or

$$
\sum_{\sigma \in \mathscr{P}_{2 k+1}}(-1)^{\sigma} \operatorname{tr}\left(\left[y, x_{\sigma(1)}\right] x_{\sigma(2)} x_{\sigma(3)} \cdots x_{\sigma(2 k)} x_{\sigma(2 k+1)}\right)=0 .
$$

It is sometimes helpful to set apart the variable of $T_{k}$ which is not alternating, so we let

$$
T_{k}\left(x_{1}, x_{2}, \ldots, x_{k-1} ; y\right)=T_{k}^{1}\left(y, x_{1}, x_{2}, \ldots, x_{k-1}\right)
$$

or

$$
T_{k}^{i}\left(x_{1}, \ldots, x_{k}\right)=(-1)^{i-1} T_{k}\left(x_{1}, \ldots, \hat{x}_{i}, \ldots, x_{k} ; x_{i}\right) .
$$

Denote by $I\left(H_{n}(F)\right)$ or $I\left(H_{n}\right)$ the ideal of identities of $H_{n}(F)$ in a free associative algebra on an at most countable number of variables (drawn from the end of the alphabet). Let $K\left(A,{ }^{*}\right)=\left\{a-a^{*} \mid a \in A\right\}$. These are the ${ }^{*}$-skew elements of $A$ if $\operatorname{char} F \neq 2$. Let $K_{n}(F)=K\left(M_{n}(F), t\right)$. If $e_{i j}$ is a set of matrix units of $M_{n}$ let $e[i j]=e_{i j}+e_{j i}, i \neq j, e[i i]=e_{i i}$. The set $\{e[i j] \mid 1 \leq i \leq j \leq n\}$ is a basis of $H_{n}$. Since $T_{2 n}$ is multilinear, it is an identity of $H_{n}$ if and only if it is zero for every substitution from a given basis of $H_{n}$. Since the above basis belongs to $H_{n}(\mathbf{Z})$ it suffices to prove $T_{2 n} \in I\left(H_{n}(F)\right)$ for $F$ of characteristic 0 .

Proposition 1. If $n$ is even, $T_{2 n}\left(x_{1}, \ldots, x_{2 n-1} ; y\right) \in I\left(H_{n}\right)$.

Proof. We may assume that $F$ is of characteristic 0 and write $n=2 m$. If $c$ is an invertible element of $K_{n}$, then the map $a^{*}=c^{-1} a^{t} c, a \in M_{n}$, defines 
an involution of symplectic type on $M_{n}$ whose symmetric elements are of the form $b c, b \in K_{n}$. (Were $c \in H_{n}$, the involution ${ }^{*}$ would be of orthogonal type.) By (1) they satisfy

$$
(b c)^{m}-\mu_{1}(b c)^{m-1}+\cdots+(-1)^{i} \mu_{i}(b c)^{m-i}+\cdots+(-1)^{m} \mu_{m}=0 .
$$

By a Zariski density argument, (7) holds for all $b, c \in K_{n}$, not only for invertible $c$ 's. Each $\mu_{i}$ is a polynomial in traces of powers of $b c$ with rational coefficients. Linearizing (7) completely we get

$$
\begin{aligned}
0= & \sum_{\sigma, \pi \in \mathscr{P}_{m}} b_{\sigma(1)} c_{\pi(1)} b_{\sigma(2)} c_{\pi(2)} \cdots b_{\sigma(m)} c_{\pi(m)} \\
& + \text { a linear combination of products of the form } b_{\sigma\left(i_{1}\right)} c_{\pi\left(i_{1}\right)} \cdots b_{\sigma\left(i_{l}\right)} c_{\pi\left(i_{l}\right)},
\end{aligned}
$$

$1 \leq i_{1} \leq i_{2}<\cdots<i_{l} \leq m, l<m$, whose coefficients are polynomials in traces of products of similar form, i.e., $b_{\sigma\left(j_{1}\right)} c_{\pi\left(j_{1}\right)} \cdots b_{\sigma\left(j_{r}\right)} c_{\pi\left(j_{r}\right)}$.

Substitute $b_{1}=\left[d, a_{1}\right], c_{1}=\left[a_{2}, a_{3}\right], b_{2}=\left[a_{4}, a_{5}\right], \ldots, c_{m}=\left[a_{2 n-2}\right.$, $\left.a_{2 n-1}\right], d, a_{i}$ 's $\in H_{n}$, so that the commutators are elements of $K_{n}$. Then sum over all permutation in $S_{2 n-1}$ multiplying each summand by the sign of the permutation. The first part of this sum is $2^{m-1} T_{2 n}\left(a_{1}, \ldots, a_{2 n-1} ; d\right)$. The remaining terms have as coefficients polynomials in traces. The traces in which $d$ does not appear have the form $\operatorname{tr}\left(S_{2 k}\left(a_{i_{1}}, \ldots, a_{i_{2 k}}\right)\right)$ which is zero by (4). If $d$ is present, since we may permute the commutators cyclically without changing the value of the trace, we may assume that $d$ is in the first commutator and we have

$$
\sum_{\sigma \in \mathscr{S}_{2 k+1}}(-1)^{\sigma} \operatorname{tr}\left(\left[d, a_{\sigma\left(i_{1}\right)}\right]\left[a_{\sigma\left(i_{2}\right)}, a_{\sigma\left(i_{3}\right)}\right] \cdots\left[a_{\sigma(2 k)}, a_{\sigma(2 k+1)}\right]\right),
$$

which is zero by (5). Thus the sum of all remaining terms is zero and

$$
T_{2 n}\left(x_{1}, \ldots, x_{2 n-1} ; y\right) \in I\left(H_{n}\right)
$$

when $n$ is even.

The following lemma will allow us to pass from $n$ even to $n$ odd.

Lemma 2. If $P\left(x_{1}, \ldots, x_{k}\right)$ is a multilinear identity of $H_{n}$, then

$$
\begin{aligned}
P\left(x_{1}, \ldots, x_{k}\right)= & P^{\prime}\left(x_{1}, \ldots, x_{k-2}\right) x_{k-1} x_{k} \\
& + \text { terms which do not end in } x_{k-1} x_{k}
\end{aligned}
$$

and $P^{\prime}\left(x_{1}, \ldots, x_{k-2}\right)$ is an identity of $H_{n-1}$.

Proof. The first half of the statement is obvious. Consider substitutions $x_{l} \in$ $\{e[i j] \mid 1 \leq i, j \leq n-1\}, 1 \leq l<k-1$ and $x_{k-1}=e[j n], x_{k}=e[n n]$. Terms in $e_{i n}$ must come from $P^{\prime}\left(x_{1}, \ldots, x_{k-2}\right) x_{k-1} x_{k}$. Since $j$ is allowed to vary, $P^{\prime}\left(x_{1}, \ldots, x_{k-2}\right)$ must be zero for every substitution from $H_{n-1}$ viewed as embedded in the upper left corner of $M_{n}$.

The same result holds for terms which begin in $x_{k} x_{k-1}$. 
If $n$ is odd, $n+1$ is even and $T_{2 n+2} \in I\left(H_{n+1}\right)$. Since

$$
\begin{aligned}
T_{2 n+2}\left(x_{1}, \ldots, x_{2 n+1} ; y\right)= & T_{2 n}\left(x_{1}, \ldots, x_{2 n-1} ; y\right) x_{2 n} x_{2 n+1} \\
& + \text { terms which do not end in } x_{2 n} x_{2 n+1},
\end{aligned}
$$

$T_{2 n}\left(x_{1}, \ldots, x_{2 n-1} ; y\right) \in I\left(H_{n}\right)$ by Lemma 2 and we have proved the first part of Theorem 1.1.

Since the polynomial $Q\left(x_{1}, x_{2}, x_{3}, x_{4}, x_{5}, x_{6}\right)$ is multilinear, we may assume that $F$ is of characteristic 0 . If $a, b, c \in K_{3}$, a simple computation shows that

$$
\{a b c\}=\frac{1}{2}(\operatorname{tr}(a b) c+\operatorname{tr}(c b) a), \quad a, b, c \in K_{3} .
$$

If $a_{i} \in H_{3}, 1 \leq i \leq 6$, then $\left[a_{i}, a_{j}\right] \in K_{3}$, and (8) may be used to express $Q\left(a_{1}, a_{2}, a_{3}, a_{4}, a_{5}, a_{6}\right)$ in terms of traces. Collecting terms and using $\left(5^{\prime}\right)$ with $k=1$, this is seen to be zero. Thus we have $Q\left(x_{1}, x_{2}, x_{3}, x_{4}, x_{5}, x_{6}\right) \in$ $I\left(H_{3}\right)$, which completes the proof of Theorem 1.1.

Standard arguments yield

Corollary 3. If $R$ is a commutative ring with unit element 1 , then

$$
T_{2 n}\left(x_{1}, \ldots, x_{2 n-1} ; y\right) \in I\left(H_{n}(R)\right) .
$$

If $A$ is a central simple associative algebra of degree $n$ over its centre and * an orthogonal involution of $A$, then $T_{2 n}\left(x_{1}, \ldots, x_{2 n-1} ; y\right) \in I\left(H\left(A,{ }^{*}\right)\right)$. If $n=3$, then $Q\left(x_{1}, x_{2}, x_{3}, x_{4}, x_{5}, x_{6}\right) \in I\left(H\left(A,{ }^{*}\right)\right)$.

We will need a few properties of $T_{n}$. By definition $T_{k}\left(x_{1}, \ldots, x_{k-1} ; y\right)$ is multilinear and it is alternating in the $x$ 's. From the definition, if $k \equiv 2,3$ $\bmod 4$,

(9) $T_{k+1}\left(x_{1}, \ldots, x_{k} ; y\right)=\sum_{i=1}^{k}(-1)^{k-i} T_{k}\left(x_{1}, \ldots, \hat{x}_{i}, \ldots, x_{k} ; y\right) x_{i}$,

$$
k \equiv 2,3 \bmod 4 \text {. }
$$

While if $k \equiv 0,1 \bmod 4$,

$$
\begin{array}{r}
T_{k+1}\left(x_{1}, \ldots, x_{k} ; y\right)=(-1)^{k} S_{k}\left(x_{1}, \ldots, x_{k}\right) y \\
+\sum_{i=1}^{k}(-1)^{k-i} T_{k}\left(x_{1}, \ldots, \hat{x}_{i}, \ldots, x_{k} ; y\right) x_{i}, \\
k \equiv 0,1 \bmod 4 .
\end{array}
$$

Lemma 4. (i) For all $j \geq 0, S_{2 n+j}, T_{2 n+j} \in I\left(H_{n}\right)$.

(ii) If any one of the variables is set equal to 1 , then $S_{2 n}$ and $T_{2 n}$ vanish.

(iii) $\sum_{i=1}^{2 n} T_{2 n}^{i}\left(x_{1}, \ldots, x_{2 n}\right)=e(n) S_{2 n}\left(x_{1}, \ldots, x_{2 n}\right)$.

(iv) If $n \geq 2$ and char $F$ does not divide $e(n)$ !, then $\left\{T_{2 n}^{i} \mid 1 \leq i \leq 2 n\right\}$ are linearly independent. 
Proof. Since $S_{2 n}$ and $T_{2 n} \in I\left(H_{n}\right)$, (i) follows from equations (3), (9), and (9') by induction on $j$. Part (ii) follows from the alternating nature of $S_{2 n}$ and $T_{2 n}$.

Consider $\sum_{j=1}^{2 n} T_{2 n}^{j}$. We claim that this polynomial is alternating. If we transpose $x_{i}$ and $x_{i+1}$, then every summand changes sign except possibly $T_{2 n}^{i}$ and $T_{2 n}^{i+1}$. Using $\left(6^{\prime}\right)$ these are $(-1)^{i-1} T_{2 n}\left(x_{1}, \ldots, \hat{x}_{i}, \ldots, x_{2 n} ; x_{i}\right)+(-1)^{i} T_{2 n}\left(x_{1}, \ldots, \hat{x}_{i+1}, \ldots, x_{2 n} ; x_{i+1}\right)$, which become

$$
\begin{gathered}
(-1)^{i-1} T_{2 n}\left(x_{1}, \ldots, x_{i-1}, x_{i}, \hat{x}_{i+1}, x_{i+2}, \ldots, x_{2 n} ; x_{i+1}\right) \\
+(-1)^{i} T_{2 n}\left(x_{1}, \ldots, x_{i-1}, \hat{x}_{i}, x_{i+1}, \ldots, x_{2 n} ; x_{i}\right)
\end{gathered}
$$

or $-\left(T_{2 n}^{i+1}+T_{2 n}^{i}\right)$. So the above sum is alternating and hence a multiple of $S_{2 n}$. Considering the monomial $x_{1} x_{2} \cdots x_{2 n}$, we see that its coefficient is $e(n)$ and we have (iii).

If char $F$ divides $e(n)$ then (iii) provides a linear dependence relation among the $T_{2 n}^{i}$. We prove (iv) by induction on $n$. If $n=2$ there are four $T_{4}^{i}$, and one checks that if $\operatorname{char} F \neq 2$ they are linearly independent. Assume that $\left\{T_{2 n}^{i} \mid 1 \leq i \leq 2 n\right\}$ are linearly independent when char $F+e(n)$ !. If $\operatorname{char} F \nmid e(n+1)$ !, then a fortiori $\operatorname{char} F \nmid e(n)$ !. By (9) and $\left(9^{\prime}\right)$, for $1 \leq i \leq 2 n$

$$
\begin{aligned}
& T_{2 n+2}^{i}\left(x_{1}, \ldots, x_{2 n+2}\right)=T_{2 n}^{i}\left(x_{1}, \ldots, x_{2 n}\right)\left[x_{2 n+1}, x_{2 n+2}\right] \\
& \quad+\text { terms which do not end in } x_{2 n+1} x_{2 n+2} \text { or } x_{2 n+2} x_{2 n+1} .
\end{aligned}
$$

Therefore $\left\{T_{2 n+2}^{i} \mid 1 \leq i \leq 2 n\right\}$ are linearly independent. If $n$ is odd then by (9), $T_{2 n+2}^{2 n+1}$ and $T_{2 n+2}^{2 n+2}$ have no terms ending in $x_{2 n+1} x_{2 n+2}$ or $x_{2 n+2} x_{2 n+1}$. In this case we need only to check the linear independence of $T_{2 n+1}^{2 n+1}$ and $T_{2 n+2}^{2 n+2}$. The induction hypothesis will do it if we consider the terms ending in $x_{1} x_{2}$ for these two polynomials.

If $n$ is even

$$
\begin{aligned}
T_{2 n+2}^{2 n+1}= & S_{2 n}\left(x_{1}, \ldots, x_{2 n}\right)\left[x_{2 n+1}, x_{2 n+2}\right] \\
& + \text { terms which do not end in } x_{2 n+1} x_{2 n+2} \text { or } x_{2 n+2} x_{2 n+1},
\end{aligned}
$$

and

$$
\begin{aligned}
T_{2 n+2}^{2 n+2}= & S_{2 n}\left(x_{1}, \ldots, x_{2 n}\right)\left[x_{2 n+1}, x_{2 n+2}\right] \\
& + \text { terms which do not end in } x_{2 n+1} x_{2 n+2} \text { or } x_{2 n+2} x_{2 n+1} .
\end{aligned}
$$

Subtracting

$$
\frac{1}{e(n)} \sum_{i=1}^{2 n} T_{2 n}^{i}\left[x_{2 n+1}, x_{2 n+2}\right]
$$

from $T_{2 n+2}^{2 n+2}$ reduces the proof to checking that these two polynomials are independent. Again considering the terms ending in $x_{1} x_{2}$ does it and we have (iv). 
The $F$-span of $\left\{T_{2 n}^{i} \mid 1 \leq i \leq 2 n\right\}$ is an $S_{2 n}$ module under the action which permutes the variables. One can check that if char $F \nmid e(n)$ ! the character of this representation is $\left[2,1^{2 n-2}\right]+\left[1^{2 n}\right]$ in the usual notation for Young diagrams.

Since $S_{2 n} \in I\left(H_{n}\right)$ irrespective of the characteristic

$$
\begin{aligned}
& S_{2 n}\left(x_{1}, \ldots, x_{2 n}\right)-T_{2 n}^{i}\left(x_{1}, \ldots, x_{2 n}\right) \\
& \quad=\sum_{\substack{\sigma \in S_{2 n} \\
\sigma^{-1}(i) \equiv 0,3 \bmod 4}}(-1)^{\sigma} x_{\sigma(1)} \cdots x_{\sigma(2 n)} \in I\left(H_{n}\right) .
\end{aligned}
$$

Just as the Amitsur-Levitzki theorem lends itself to a graph-theoretic interpretation and is equivalent to a result on unicursal (or Eulerian) paths on a directed graph [2, p. 232; 11 , Theorem 2], Theorem 1.1 is equivalent to a result on unicursal paths on undirected graphs. We still state this for the first part of the theorem.

Let $(V, E)$ be a finite graph with vertices $V=\left\{v_{1}, \ldots, v_{n}\right\}$ and (undirected) edges $E=\left\{w_{1}, \ldots, w_{r}\right\}$ in an arbitrary but fixed ordering. Then unicursal paths (if they exist) correspond to permutations $\sigma \in \mathscr{S}_{r}$, where the path is $w_{\sigma(1)} w_{\sigma(2)} \cdots w_{\sigma(r)}=p_{\sigma}$. Define $\varepsilon\left(p_{\sigma}\right)$ to be $(-1)^{\sigma}$.

Theorem 5. Let $(V, E)$ be an undirected graph with $|E| \geq 2|V|$. Choose a distinguished edge $w_{i}$. Then for any two fixed vertices $v_{j}, v_{k}$ (not necessarily distinct) the number of unicursal paths $p_{\sigma}$ from $v_{j}$ to $v_{k}$ with $w_{i}$ in positions which are congruent to 1 or 2 modulo 4 and with $\varepsilon\left(p_{\sigma}\right)=1$ is equal to the number of such paths with $\varepsilon\left(p_{\sigma}\right)=-1$. The same holds for unicursal paths with $w_{i}$ in positions which are congruent to 0 or 3 modulo 4.

It is easy to see the equivalence if we identify an edge joining $v_{i}$ and $v_{j}$ to $e[i j]=e[j i] \quad(i$ may equal $j)$ and note that unicursal paths correspond to nonzero monomials.

Since $T_{2 n}$ is not alternating, putting two properly chosen variables equal will yield a polynomial of degree 2 in that variable. This polynomial, as expected, will play a role in the proof of Theorem 2 . For $i, j, k$ with $1 \leq i \leq j-1 \leq k-2$ define

$$
\begin{aligned}
& G_{k}^{i j}\left(x_{1}, \ldots, x_{k-2} ; y\right) \\
&=\sum_{\sigma \in \mathscr{P}_{k-2}}(-1)^{\sigma} x_{\sigma(1)} \cdots x_{\sigma(i-1)}\left[y, x_{\sigma(i)}\right] x_{\sigma(i+1)}, \ldots, \\
& x_{\sigma(j-2)}\left[y, x_{\sigma(j-1)}\right] x_{\sigma(j)} \cdots x_{\sigma(k-2)},
\end{aligned}
$$

$$
\begin{aligned}
G_{k}\left(x_{1}, \ldots, x_{k-2} ; y\right) & \\
& =\sum_{j=0}^{[k / 4-1]} \sum_{i=1}^{[k / 2-2 j-1]}(-1)^{i-1} G_{k}^{2 i-12 i-1+4 j+2}\left(x_{1}, \ldots, x_{k-2} ; y\right) .
\end{aligned}
$$


Proposition 6. $T_{2 n}\left(x_{1}, \ldots, x_{2 n-2}, y ; y\right)=-G_{2 n}\left(x_{1}, \ldots, x_{2 n-2} ; y\right)$ and therefore $G_{2 n}$ belongs to $I\left(H_{n}\right)$.

Proof. A typical expression of $T_{2 n}\left(x_{1}, \ldots, x_{2 n-2}, y ; y\right)$ is of the form

$$
x_{\sigma(1)} \cdots x_{\sigma(i-1)}\left[y, x_{\sigma(i)}\right] x_{\sigma(i+1)} \cdots x_{\sigma(j-2)}\left[y, x_{\sigma(j-1)}\right] x_{\sigma(j)} \cdots x_{\sigma(2 n-2)} .
$$

To avoid counting the same monomial twice, we may assume that $i$ and $j$ are odd. Such an expression may arise from

$$
x_{\sigma(1)} \cdots x_{\sigma(i-1)}\left[y, x_{\sigma(i)}\right] x_{\sigma(i+1)} \cdots x_{\sigma(j-2)}\left[x_{\sigma(2 n-1)}, x_{\sigma(j-1)}\right] x_{\sigma(j)} \cdots x_{\sigma(2 n-2)}
$$

if $i \equiv 1 \bmod 4$; in this case its sign is $(-1)^{j}(-1)^{\sigma}=-(-1)^{\sigma}$ since $j$ is odd. Or it may arise from

$$
x_{\sigma(1)} \cdots x_{\sigma(i-1)}\left[x_{\sigma(2 n-1)}, x_{\sigma(i)}\right] x_{\sigma(i+1)} \cdots x_{\sigma(j-2)}\left[y, x_{\sigma(j-1)}\right] x_{\sigma(j)} \cdots x_{\sigma(2 n-2)}
$$

if $j \equiv 1 \bmod 4$; in this case the sign is $(-1)^{i-1}(-1)^{\sigma}=(-1)^{\sigma}$ since $i$ is odd. Both will occur if $i \equiv 1 \bmod 4$ and $j \equiv 1 \bmod 4$ in which case they cancel since they have opposite signs. So we are left with (16) with $i, j$ odd and exactly one index is congruent to $1 \bmod 4$. If $i \equiv 1$ and $j \equiv 3$ or $i \equiv 3$ and $j \equiv 1(\bmod 4)$ their difference is congruent to $2 \bmod 4$. So we get exactly the same terms as are present in $G_{2 n}$ and we must check that they have opposite signs. If $i \equiv 1$ and $j \equiv 3$ the sign is $-(-1)^{\sigma}$ while when $i=3$ the sign is $(-1)^{\sigma}$. Writing $i=2 i^{\prime}-1$ we get $-(-1)^{\sigma}$ when $i^{\prime}$ is odd and $(-1)^{\sigma}$ when $i^{\prime}$ is even. Comparing with $(15)$ we see that $T_{2 n}\left(x_{1}, \ldots, x_{2 n-2} ; y ; y\right)$ and $G_{2 n}\left(x_{1}, \ldots, x_{2 n-2} ; y\right)$ have opposite signs.

The polynomial $Q\left(x_{1}, x_{2}, x_{3}, x_{4}, x_{5}, x_{6}\right)$ is skew in $x_{1}, x_{2}, x_{3}$ and $x_{4}$, $x_{5}, x_{6}$. Moreover

$$
Q\left(x_{4}, x_{5}, x_{6}, x_{1}, x_{2}, x_{3}\right)=-Q\left(x_{1}, x_{2}, x_{3}, x_{4}, x_{5}, x_{6}\right) \text {. }
$$

This can be used to show that if $\operatorname{char} F \neq 2$ then the $F$-span of

$$
Q\left(x_{\sigma(1)}, x_{\sigma(2)}, \ldots, x_{\sigma(6)}\right),
$$

$\sigma \in \mathscr{S}_{6}$, has dimension 10 and its character as an $\mathscr{S}_{6}$-module is $\left[2^{2}, 1^{2}\right]+\left[1^{6}\right]$. Let

$$
\begin{gathered}
Q\left(x_{1}, x_{2}, x_{3}, x_{4} ; y\right)=Q\left(x_{1}, x_{2}, y, x_{3}, x_{4}, y\right), \\
Q\left(x_{1}, x_{2} ; y, z\right)=Q\left(x_{1}, z, x_{2}, z ; y\right) .
\end{gathered}
$$

The polynomial $Q\left(x_{1}, x_{2}, x_{3}, x_{4} ; y\right)$ is $x_{1}, x_{2}$ skew, $x_{3}, x_{4}$ skew and

$$
Q\left(x_{3}, x_{4}, x_{1}, x_{2} ; y\right)=-Q\left(x_{1}, x_{2}, x_{3}, x_{4} ; y\right) \text {. }
$$

This is enough to prove that the $F$-span of $Q\left(x_{\sigma(1)}, x_{\sigma(2)}, x_{\sigma(3)}, x_{\sigma(4)} ; y\right)$, $\sigma \in \mathscr{S}_{4}$, is the $F$-span of

$$
Q\left(x_{1}, x_{2}, x_{3}, x_{4} ; y\right), \quad Q\left(x_{1}, x_{3}, x_{4}, x_{2} ; y\right), \quad \text { and } Q\left(x_{1}, x_{4}, x_{2}, x_{3} ; y\right) \text {. }
$$


In fact one can check that these three polynomials and $G_{6}\left(x_{1}, x_{2}, x_{3}, x_{4} ; y\right)$ are linearly independent.

\section{UNIQUENESS}

Essentially Theorem 1.2 says that, under some restrictions on the base field and if $n \neq 3$, any homogeneous polynomial identity of $H_{n}$ of degree $2 n$ is obtained from $T_{2 n}$. To prove that, we must substitute values of $H_{n}$ for the variables and conclude that some polynomials are not identities. While we try to be as systematic as possible, the need to include $Q$ when $n=3$ forces us to consider low $n$ 's very carefully.

We recall a few standard facts concerning polynomial identities stating them for $H_{n}$. If $H_{n}(F)$ satisfies a polynomial identity, then it satisfies a multilinear identity of the same degree. If $P\left(x_{1}, \ldots, x_{m}\right)$ is a homogeneous identity of $H_{n}(F)$ then its linearization in all but one variable is an identity of the same total degree and of the same degree as $P$ in the nonlinearized variable. If $P\left(x_{1}, \ldots, x_{m}, y\right)$ is a homogeneous polynomial, linear in the $x$ 's and of degree $k$ in $y$, then the polynomial obtained by first linearizing $P$ completely then replacing the new variables by $y$ is $k ! P\left(x_{1}, \ldots, x_{m}, y\right)$. Therefore if $\operatorname{char} F \nmid k !, P\left(x_{1}, \ldots, x_{m}, y\right) \in I\left(H_{n}\right)$ if and only if the total linearization of $P \in I\left(H_{n}\right)$. One advantage in dealing with multilinear identities is that they remain identities when the base field is extended. Finally if the degree of each $x_{i}$ in an identity $P\left(x_{1}, \ldots, x_{m}\right)$ is less than $|F|$ then each homogeneous component of $P$ is also an identity.

Following Osborn [4, p. 78] we introduce a partial ordering on the set of homogeneous polynomials in noncommuting indeterminates $x_{1}, \ldots, x_{m}$. If $P\left(x_{1}, \ldots, x_{m}\right)$ is a homogeneous polynomial of degree $n$, we say that it is of type $\left[n_{1}, n_{2}, \ldots, n_{m}\right]$ if $n_{j}$ is the degree of $x_{j}$ in $P$ and $n_{1} \geq n_{2} \geq$ $\cdots \geq n_{m}$. If $P$ and $P^{\prime}$ are homogeneous of degree $n$ and $n^{\prime}$ and of type $\left[n_{1}, n_{2}, \ldots, n_{m}\right]$ and $\left[n_{1}^{\prime}, n_{2}^{\prime}, \ldots, n_{m}^{\prime}\right]$ respectively, then $P$ is lower than $P^{\prime}$ in the partial ordering if and only if either (i) $n<n^{\prime}$ or (ii) $n=n^{\prime}$ and $n_{j}>n_{j}^{\prime}$ for the first integer $j$ such that $n_{j} \neq n_{j}^{\prime}$. Otherwise two polynomials are not comparable. If an integer is repeated $k$ times, we will denote this by an exponent. For example $\left[3,2^{2}, 1^{3}\right]$ means $[3,2,2,1,1,1]$.

An identity is absolutely irreducible if it does not imply an identity lower than itself in the partial ordering. While the following theorem is proved in [4, Theorem 3] for elements of the free nonassociative algebra, the author notes that essentially the same proof will work in the free associative algebra.

Theorem (Osborn). Let $n$ be a positive integer and $P\left(x_{1}, \ldots, x_{m}\right)$ an absolutely irreducible homogeneous polynomial with coefficients in a field $F$ of characteristic not dividing $n$ !. Then $P$ is either symmetric or skew-symmetric in its arguments of degree $n$, depending on whether $n$ is even or odd.

Let ${ }^{*}$ denote the unique involution on a free associative algebra which restricts to the identity on the generators. A polynomial $P$ is said to be symmetric 
if $P^{*}=P$, skew-symmetric if $P^{*}=-P$. If $P$ is multilinear of degree $m$,

$$
P\left(x_{1}, x_{2}, \ldots, x_{m}\right)=\sum_{\sigma \in \mathscr{S}_{m}} \alpha_{\sigma} x_{\sigma(1)} x_{\sigma(2)} \cdots x_{\sigma(m)},
$$

then

$$
P\left(x_{1}, x_{2}, \ldots, x_{m}\right)^{*}=\sum_{\sigma \in \mathscr{S}_{m}} \alpha_{\rho \sigma} x_{\sigma(1)} x_{\sigma(2)} \cdots x_{\sigma(m)},
$$

where $\rho$ is the reversal permutation

$$
(1 m)(2 m-1) \cdots\left(\left[\frac{m}{2}\right]\left[\frac{m+1}{2}\right]+1\right) .
$$

We multiply permutations from right to left. The sign of $\rho$ is 1 if $m$ is congruent to 0 or $1 \bmod 4,-1$ if $m$ is congruent to 2 or $3 \bmod 4$. Taking the transpose of any evaluation we see that if $P \in I\left(M_{n}\right)$ or $I\left(H_{n}\right)$ then so does $P^{*}$. If the characteristic is not 2 then any identity is the sum of its symmetric and skew-symmetric parts and these are also identities.

The uniqueness part of the Amitsur-Levitzki theorem is proved using two types of substitutions. The first corresponds to

Lemma 1. If $P\left(x_{1}, \ldots, x_{2 n}\right)=\sum_{\sigma \in \mathscr{S}_{2 n}} \alpha_{\sigma} x_{\sigma(1)} \cdots x_{\sigma(2 n)}$ is a multilinear identity of $H_{n}$, then

$$
\alpha_{\sigma(2 i-12 i)}=-\alpha_{\sigma}, \quad 1 \leq i \leq n .
$$

If $P$ is a homogeneous identity of $H_{n}$ of degree $2 n$, then the words which differ only by a transposition of the indeterminates in positions $2 i-1$ and $2 i$ have coefficients which differ only in sign. In particular if char $F \neq 2$ the words having the same indeterminate in positions $2 i-1$ and $2 i$, have coefficient 0 in $P$.

Proof. Substituting $e[11], e[12], e[22], e[23], \ldots, e[i-1 i-1], e[i-1 i]$, $e[i i], e[i i], e[i i+1], e[i+1 i+1], \ldots, e[n-1 n], e[n n]$ for $x_{\sigma(1)}, x_{\sigma(2)}, x_{\sigma(3)}$, $x_{\sigma(4)}, \ldots, x_{\sigma(2 i-3)}, x_{\sigma(2 i-2)}, x_{\sigma(2 i-1)}, x_{\sigma(2 i)}, x_{\sigma(2 i+1)}, x_{\sigma(2 i+2)}, \ldots, x_{\sigma(2 n)}$ in $P$ and considering the coefficient of $e_{1 n}$ yields the first result. The second part is obtained by linearizing and applying the first result.

From now on we assume that $F$ is a field of characteristic not 2 .

Corollary 2. Any homogeneous identity $P$ of $H_{n}$ of degree $2 n$ is a linear combination of associative products of commutators. In particular no variable has degree greater than $n$.

Since Jordan polynomials are symmetric, identities which are symmetric are of particular interest.

Lemma 3. If $P\left(x_{1}, \ldots, x_{2 n}\right)=\sum_{\sigma \in \mathscr{P}_{2 n}} \alpha_{\sigma} x_{\sigma(1)} \cdots x_{\sigma(2 n)}$ is a multilinear identity of $H_{n}$, then

$$
\alpha_{\sigma}+\alpha_{\rho \sigma}+\alpha_{\sigma(2 i 2 i+1)}+\alpha_{\rho \sigma(2 i 2 i+1)}=0, \quad 1 \leq i \leq n-1 .
$$


In particular if $P$ is symmetric then

Proof. Substituting

$$
\alpha_{\sigma(2 i 2 i+1)}=-\alpha_{\sigma}, \quad 1 \leq i \leq n-1 .
$$

$$
\begin{gathered}
e[12], e[22], \ldots, e[i-1 i], e[i i], e[i i], e[i i+1], \\
e[i+1 i+1], e[i+1 i+2], \ldots, e[n n] e[1 n]
\end{gathered}
$$

for $x_{\sigma(1)}, x_{\sigma(2)}, \ldots, x_{\sigma(2 i-1)}, x_{\sigma(2 i)}, x_{\sigma(2 i+1)}, x_{\sigma(2 i+2)}, x_{\sigma(2 i+3)}, x_{\sigma(2 i+4)}, \ldots$, $x_{\sigma(2 n-1)}, x_{\sigma(2 n)}$ in $P$ and considering the coefficient of $e_{11}$ yields the first result. If $P$ is symmetric, then $\alpha_{\sigma}=\alpha_{\rho \sigma}$ for all $\sigma$ and since char $F \neq 2$ the second equality holds.

If $P$ is a symmetry identity of $H_{n}$ homogeneous of degree $2 n$, then, by Lemmas 1 and 3, its complete linearization is a multiple of $S_{2 n}$. Hence $P$ was multilinear to start with. Considering the sign of $\rho, S_{2 n}$ is symmetric if $n$ is even, skew-symmetric if $n$ is odd and we have

Corollary 4. If $n$ is odd then $H_{n}$ has no symmetric homogeneous identity of degree $2 n$. If $n$ is even, the only symmetric homogeneous identities of $H_{n}$ of degree $2 n$ are multiples of $S_{2 n}$. Thus any homogeneous identity of $H_{n}$ of degree $2 n$ of lower type than $\left[1^{2 n}\right]$ must be skew-symmetric.

Since Smith [10, Corollary 2] has shown that the standard polynomial is not Jordan if char $F \nmid(2 n)$ !, in this case $H_{n}$ does not satisfy any Jordan polynomial of degree $2 n$.

Lemma 5. Let $P\left(x_{1}, \ldots, x_{2 n-1} ; y\right)$ be an identity of $H_{n}$ of type $\left[2,1^{2 n-2}\right]$. Then we may write

$$
\begin{array}{r}
P\left(x_{1}, \ldots, x_{2 n-2} ; y\right) \\
=\sum_{1 \leq i \leq j \leq n-1} \sum_{\sigma \in \mathscr{P}_{2 n-2}} \alpha_{\sigma}^{i j}\left[x_{\sigma(1)}, x_{\sigma(2)}\right] \cdots\left[y, x_{\sigma(2 i-1)}\right]\left[x_{\sigma(2 i)}, x_{\sigma(2 i+1)}\right] \\
\cdots\left[y, x_{\sigma(2 j)}\right]\left[x_{\sigma(2 j+1)}, x_{\sigma(2 j+2)}\right] \cdots\left[x_{\sigma(2 n-3)}, x_{\sigma(2 n-2)}\right],
\end{array}
$$

and

$$
\alpha_{\sigma}^{i i}=-\alpha_{\sigma(2 i-12 i)}^{i i} .
$$

Hence a homogeneous identity of $H_{n}$ of degree $2 n$ cannot have terms

$$
\cdots[y, x][y, x] \cdots
$$

with nonzero coefficient.

Proof. By Corollary 2 we may write $P$ as above. Consider the coefficient of $e_{1 n}$ when $e[11], e[12], \ldots, e[i i], e[i+1 i+1], e[i+2 i+2], e[i i+2], \ldots, e[n n]$, $e[n-1 n]$ are substituted for $x_{\sigma(1)}, x_{\sigma(2)}, \ldots, x_{\sigma(2 i-1)}, x_{\sigma(2 i)}, x_{\sigma(2 i+1)}, x_{\sigma(2 i+2)}$, $\ldots, x_{\sigma(2 n-3)}, x_{\sigma(2 n-2)}$ and $e[i i+1]$ for $y$ in $P$. Since it is $\left(\alpha_{\sigma}^{i i}-\alpha_{\sigma(2 i-12 i)}^{i i}\right)$ we have $\alpha_{\sigma}^{i i}=-\alpha_{\sigma(2 i-12 i)}^{i i}$. The last part is obtained by partial linearization.

We are now ready to consider the case $n=2$. 
Proposition 6. If char $F \neq 2$ and $|F|>3$, then the identities of minimal degree of $\mathrm{H}_{2}(F)$ are consequences of $T_{4}\left(x_{1}, x_{2}, x_{3} ; y\right)$.

Proof. Since the base field has more than 4 elements we need only consider homogeneous polynomials. By Lemma 2.4(iii), since $\operatorname{char} F \neq 2, S_{4}$ is a consequence of $T_{4}$. Moreover since

$$
\sum_{i=1}^{4} T_{4}\left(x_{1}, \ldots, \hat{x}_{i}, \ldots, x_{4} ; x_{i}\right)=2\left[\left[x_{2}, x_{4}\right],\left[x_{1}, x_{3}\right]\right],
$$

$\left[\left[x_{1}, x_{2}\right],\left[x_{3}, x_{4}\right]\right]$ and hence $\left[\left[y, x_{1}\right],\left[y, x_{2}\right]\right]$ are consequences of $T_{4}$.

In the case $n=2$, Proposition 2.6 becomes

$$
T_{4}\left(x_{1}, x_{2}, y ; y\right)=-\left[\left[y, x_{1}\right],\left[y, x_{2}\right]\right] .
$$

We prefer obtaining $\left[\left[y, x_{1}\right],\left[y, x_{2}\right]\right]$ this way because $T_{A}\left(x_{1}, x_{2}, x_{3} ; y\right)$ is alternating in the $x$ 's.

If $P \in I\left(H_{2}\right)$ is homogeneous of degree 4 then it could be of type [4], [3, 1], $\left[2^{2}\right],\left[2,1^{2}\right]$ and $\left[1^{4}\right]$. By Corollary 2 the first two types cannot occur and an identity of type $\left[2^{2}\right]$ must be of the form

$$
\alpha[x, y]^{2}, \quad \alpha \neq 0,
$$

which is not an identity either directly or by Lemma 5 .

If $P\left(x_{1}, x_{2} ; y\right)$ is an identity of type $\left[2,1^{2}\right]$ then, by Corollary 2 and Lemma 5 , it must be a multiple of $\left[y, x_{1}\right]\left[y, x_{2}\right]-\left[y, x_{2}\right]\left[y, x_{1}\right]=\left[\left[y, x_{1}\right]\right.$, $\left.\left[y, x_{2}\right]\right]$.

If $P\left(x_{1}, x_{2}, x_{3}, x_{4}\right) \in I\left(H_{2}\right)$ is multilinear, then $P\left(y, y, x_{3}, x_{4}\right)$ must be a multiple of $\left[\left[y, x_{3}\right],\left[y, x_{4}\right]\right]$. Subtracting a multiple of $\left[\left[x_{1}, x_{3}\right],\left[x_{2}, x_{4}\right]\right]$ if necessary, we may assume that $P$ is skew in $x_{1}, x_{2}$. Consider next $P\left(x_{1}, y, y, x_{4}\right)$. It is a multiple of $\left[\left[y, x_{1}\right],\left[y, x_{4}\right]\right]$. Subtracting a multiple of $T_{4}\left(x_{1}, x_{2}, x_{4} ; x_{3}\right)$ if necessary, we may assume that $P\left(x_{1}, y, y, x_{4}\right)$ is identically zero and hence that $P\left(x_{1}, x_{2}, x_{3}, x_{4}\right)$ is skew in $x_{2}, x_{3}$ without losing the skewness in $x_{1}, x_{2}$. Thus we have $P$ alternating in $x_{1}, x_{2}, x_{3}$. Finally $P\left(x_{1}, x_{2}, y, y\right)$ is a multiple of $\left[\left[y, x_{1}\right],\left[y, x_{2}\right]\right]$. Subtracting a multiple of $T_{4}\left(x_{1}, x_{2}, x_{3} ; x_{4}\right)$, we may assume that $P$ is skew in $x_{3}, x_{4}$ and still alternating in $x_{1}, x_{2}, x_{3}$. Hence $P$ is alternating in all $x$ 's and a multiple of $S_{4}$. Since $S_{4}$ is a consequence of $T_{4}$ this proves the proposition.

We have given this last argument in detail because analogous ones will be used in the next case.

Remark. If $F$ is an infinite field and $P\left(x_{1}, \ldots, x_{m} ; y\right)$ is a homogeneous polynomial of type $\left[k, 1^{m}\right]$ then to show that $P$ is an identity of $H_{n}$ it suffices, by a Zariski density argument, to prove that $P$ is zero whenever $y$ is diagonal with distinct eigenvalues and the $x$ 's belong to a fixed basis of $H_{n}$. If $\operatorname{char} F \nmid$ $k$ ! then a field extension argument allows us to drop the hypothesis $F$ infinite. 
Lemma 7. Let $P\left(x_{1}, \ldots, x_{m} ; y\right)$ be an identity of $H_{n}(F), n>1$, of type $\left[k, 1^{m}\right]$ which is a linear combination of terms ending in $[y$,$] . Then$

$$
P\left(x_{1}, \ldots, x_{m} ; y\right)=\sum_{i=1}^{m} P_{i}\left(x_{1}, \ldots, \hat{x}_{i}, \ldots, x_{m} ; y\right)\left[y, x_{i}\right]
$$

If $F$ is infinite or if char $F+k$ ! then $P_{i}\left(x_{1}, \ldots, \hat{x}_{i}, \ldots, x_{m} ; y\right)$ is an identity of $H_{n-1}$ of type $\left[k-1,1^{m-1}\right]$.

Proof. By the remark we may assume that $F$ is infinite. We need only show that the $P_{i}$ 's are identities of $H_{n-1}$. Fix $i, 1 \leq i \leq m$. Let $y=\sum_{r=1}^{n} \lambda_{r} e_{r} ; \lambda_{r}$ distinct, $x_{i}=e[j n]$ for an arbitrary but fixed $j, 1 \leq j<n$, and $x_{s} \in\{e[p q] \mid 1 \leq$ $p, q<n\}, 1 \leq s \leq m, s \neq i$. Since $\left[y, x_{i}\right]=\left(\lambda_{j}-\lambda_{n}\right)\left(e_{j n}-e_{n j}\right) \neq 0$, the coefficient of the matrix units $e_{t n}, 1 \leq t<n$, in $P\left(x_{1}, \ldots, x_{m} ; y\right)$ must come from $P_{i}\left(x_{1}, \ldots, \hat{x}_{i}, \ldots, x_{m} ; y\right)$. Since $j$ is arbitrary, $P_{i}$ must vanish on $H_{n-1}$ embedded in the upper left-hand corner of $H_{n}$. So $P_{i} \in I\left(H_{n-1}\right)$.

Corollary 8. Assume that $\operatorname{char} F \nmid k !, k>1$. If $H_{n-1}$ has no identity of type $\left[k-1,1^{2 n-k-1}\right]$ then $H_{n}$ has no identity of type $\left[k, 1^{2 n-k}\right]$.

Proof. If $P\left(x_{1}, \ldots, x_{2 n-k} ; y\right) \in I\left(H_{n}\right)$ is of type $\left[k, 1^{2 n-k}\right]$ then, by Lemma 2.2, $P$ is a sum of terms ending in $[y$,$] . Then Lemma 7$ yields the result.

Proposition 9. Assume that $n>1$ and char $F \nmid n$ !. Then $H_{n}(F)$ has no identity of degree $2 n$ of type $\left[3,1^{2 n-3}\right]$ or lower.

Proof. The proof is by induction on $n$. We already have the result for $n=2$. Assume that for some $n>2, H_{n-1}$ has no identity of type $\left[3,1^{2 n-5}\right]$. Then, by Corollary $8, H_{n}$ also has no identity of type $\left[4,1^{2 n-4}\right]$ or lower.

It remains to show that $H_{n}$ has no identity of type $\left[3,1^{2 n-3}\right]$. Let

$$
P\left(x_{1}, \ldots, x_{2 n-3} ; y\right) \in I\left(H_{n}\right)
$$

be of type $\left[3,1^{2 n-3}\right]$. By Lemma 2.2 used on both sides and the induction hypothesis, none of the summands of $P$ start or end in $x_{i} x_{j}$. Therefore every summand starts and ends in $[y$,$] . So$

$$
\begin{aligned}
P\left(x_{1}, \ldots, x_{2 n-3} ; y\right)=\sum_{i=1}^{n-2} \sum_{\sigma \in \mathscr{P}_{2 n-3}} \alpha_{\sigma}^{i}\left[y, x_{\sigma(1)}\right]\left[x_{\sigma(2)}, x_{\sigma(3)}\right] \\
\\
\quad \cdots\left[y, x_{\sigma(2 i)}\right]\left[x_{\sigma(2 i+1)}, x_{\sigma(2 i+2)}\right] \cdots\left[y, x_{\sigma(2 n-3)}\right] .
\end{aligned}
$$


Since $P^{*}=-P$,

$$
\begin{aligned}
& P\left(x_{1}, \ldots, x_{2 n-3} ; y\right) \\
& =\sum_{i=1}^{[(n-2) / 2]} \sum_{\sigma \in \mathscr{S}_{2 n-3}} \alpha_{\sigma}^{i}\left(\left[y, x_{\sigma(1)}\right] \cdots\left[y, x_{\sigma(2 i)}\right] \cdots\left[y, x_{\sigma(2 n-3)}\right]\right. \\
& \left.\quad+\left[y, x_{\sigma(2 n-3)}\right] \cdots\left[y, x_{\sigma(2 i)}\right] \cdots\left[y, x_{\sigma(1)}\right]\right) \\
& \quad+\sum_{\sigma \in \mathscr{P}_{2 n-3}} \beta_{\sigma}\left[y, x_{\sigma(1)}\right] \cdots\left[x_{\sigma(n-3)}, x_{\sigma(n-2)}\right]\left[y, x_{\sigma(n-1)}\right] \cdots\left[y, x_{\sigma(2 n-3)}\right],
\end{aligned}
$$

the last term occurring only if $n$ is odd.

We show first that $\alpha_{\sigma}^{1}=0$ for all $\sigma$, then that $\alpha_{\sigma}^{i}=0$ implies $\alpha_{\sigma}^{i+1}=0$ and finally that for $n$ odd, $\beta_{\sigma}=0$.

For $\sigma \in \mathscr{S}_{2 n-3}$ let $y=e[1 n], x_{\sigma(1)}=e[2 n], x_{\sigma(2)}=e[22], x_{\sigma(3)}=$ $e[23], x_{\sigma(4)}=e[33], x_{\sigma(5)}=e[34], \ldots, x_{\sigma(2 n-6)}=e[n-2 n-2], x_{\sigma(2 n-5)}=$ $e[n-2 n-1], x_{\sigma(2 n-4)}=e[1 n-1], x_{\sigma(2 n-3)}=e[12]$. For this substitution, $\left[y, x_{\sigma(j)}\right]=0$ except $\left[y, x_{\sigma(1)}\right]=e_{12}-e_{21},\left[y, x_{\sigma(2 n-4)}\right]=e_{n n-1}-e_{n-1 n}$ and $\left[y, x_{\sigma(2 n-3)}\right]=e_{n 2}-e_{2 n}$. For the remaining $x$ 's, $x_{\sigma(j)}$ has a nonzero commutator only with $x_{\sigma(j-1)}$ and $x_{\sigma(j+1)}$. Since $x_{\sigma(1)}, x_{\sigma(2 n-4)}$ and $x_{\sigma(2 n-3)}$ are already accounted for, we must have $\left[x_{\sigma(2)}, x_{\sigma(3)}\right],\left[x_{\sigma(4)}, x_{\sigma(5)}\right], \ldots,\left[x_{\sigma(2 n-6)}\right.$, $\left.x_{\sigma(2 n-5)}\right]$. For $1 \leq j \leq n-3,\left[x_{\sigma(2 j)}, x_{\sigma(2 j+1)}\right]=e_{j+1 j+2}-e_{j+2 j+1}$. Therefore the only way to obtain $e_{12}$ is to take the product

$$
\left[y, x_{\sigma(1)}\right]\left[x_{\sigma(2)}, x_{\sigma(3)}\right] \cdots\left[x_{\sigma(2 n-6)}, x_{\sigma(2 n-5)}\right]\left[y, x_{\sigma(2 n-4)}\right]\left[y, x_{\sigma(2 n-3)}\right]
$$

and $\alpha_{\sigma}^{1}=0$.

Assume $\alpha_{\sigma}^{i}=0$ for all $\sigma \in \mathscr{S}_{2 n-3}$. For a fixed $\sigma \in \mathscr{S}_{2 n-3}$, let $x_{\sigma(2 i)}=y$ and consider the coefficient of

$$
\begin{aligned}
{\left[y, x_{\sigma(1)}\right]\left[x_{\sigma(2)},\right.} & \left.x_{\sigma(3)}\right] \\
\cdots & {\left[y, x_{\sigma(2 i+1)}\right]\left[y, x_{\sigma(2 i+2)}\right]\left[x_{\sigma(2 i+3)}, x_{\sigma(2 i+4)}\right] \cdots\left[y, x_{\sigma(2 n-3)}\right] . }
\end{aligned}
$$

It is $\alpha_{\sigma}^{i+1}+\alpha_{\sigma(2 i 2 i+1)}^{i}=\alpha_{\sigma}^{i+1}$ since $\alpha_{\sigma}^{i}=0$. Since $H_{n}$ has no identity of type $\left[4,1^{2 n-4}\right], P$ with $x_{\sigma(2 i)}$ replaced by $y$ is the zero polynomial and $\alpha_{\sigma}^{i+1}=0$. So all $\alpha_{\sigma}^{j}$ 's are 0 . The same argument using $x_{\sigma(n-3)}=y$ will yield $\beta_{\sigma}=0$ in case $n$ is odd. So $H_{n}$ has no identity of type $\left[3,1^{2 n-3}\right]$.

Proposition 10. If char $F \neq 2,3$ then any homogeneous identity of $H_{3}(F)$ of degree 6 is a consequence of $T_{6}$ and $Q$.

Proof. By Proposition 9, $\mathrm{H}_{3}$ has no identity of type $\left[3,1^{3}\right]$ or lower. By Corollary 2 and Lemma 5 , an identity of type $\left[2^{3}\right]$ must be a multiple of $S_{3}([x, y],[x, z],[y, z])$. This is a central polynomial [7, Remark 2.5.15] but not an identity. So $H_{3}$ has no identity of type $\left[2^{3}\right]$. 
Let $P\left(x_{1}, x_{2} ; y, z\right)$ be an identity of $H_{3}$ linear in $x_{1}, x_{2}$ and of degree 2 in $y$ and $z$. Since $H_{3}$ has no identity of type $\left[2^{3}\right], P(x, x ; y, z)$ is the zero polynomial and $P$ is skew in $x_{1}, x_{2}$. By Corollary $2, P$ is a linear combination of products of commutators. The only commutator which could appear twice in the same products is $[y, z]$ and by Lemma 5 these can not be adjacent. By Corollary $4, P^{*}=-P$ so we have a linear combination of terms of the form $\{[],[],[]$,$\} . Putting all this together using Lemma 5, we have$

$$
\begin{aligned}
& P\left(x_{1}, x_{2} ; y, z\right)=\alpha\left(\left\{[y, z]\left[y, x_{1}\right]\left[z, x_{2}\right]\right\}-\left\{[y, z]\left[y, x_{2}\right]\left[z, x_{1}\right]\right\}\right. \\
&-\left\{\left[y, x_{1}\right][y, z]\left[z, x_{2}\right]\right\}+\left\{\left[y, x_{2}\right][y, z]\left[z, x_{1}\right]\right\} \\
&\left.+\left\{[y, z]\left[z, x_{2}\right]\left[y, x_{1}\right]\right\}-\left\{[y, z]\left[z, x_{1}\right]\left[y, x_{2}\right]\right\}\right) \\
&+ \beta\left\{[y, z]\left[x_{1}, x_{2}\right][y, z]\right\} .
\end{aligned}
$$

Since $H_{3}$ has no identity of type $[3,2,1], P\left(x_{1}, y ; y, z\right)$ is identically zero. This yields $\alpha=\beta$. Thus $P\left(x_{1}, x_{2} ; y, z\right)$ is a multiple of $Q\left(x_{1}, x_{2} ; y, z\right)$.

Let $P\left(x_{1}, x_{2}, x_{3}, x_{4} ; y\right) \in I\left(H_{3}\right)$ be of type $\left[2,1^{4}\right]$. Since

$$
P\left(z, z, x_{3}, x_{4} ; y\right) \in I\left(H_{3}\right)
$$

is of type $\left[2^{2}, 1^{2}\right]$ it is a scalar multiple $Q\left(x_{3}, x_{4} ; y, z\right)$. So subtracting a multiple of $Q\left(x_{1}, x_{2}, x_{3}, x_{4} ; y\right)$ if necessary, we may assume that $P$ is skew in $x_{1}, x_{2}$. Similarly $P\left(x_{1}, z, z, x_{4} ; y\right)$ is a multiple of $Q\left(x_{1}, x_{4} ; y, z\right)$. Subtracting a multiple of $Q\left(x_{1}, x_{2}, x_{3}, x_{4} ; y\right)$ if necessary, we may assume that $P$ is skew in $x_{2}, x_{3}$ without losing the fact that it is skew in $x_{1}, x_{2}$. Finally $P\left(x_{1}, x_{2}, z, z ; y\right)$ is a multiple of $Q\left(x_{1}, x_{2} ; y, z\right)$. The polynomial

$$
Q\left(x_{1}, x_{2}, x_{3}, x_{4} ; y\right)+Q\left(x_{2}, x_{3}, x_{1}, x_{4} ; y\right)+Q\left(x_{3}, x_{1}, x_{2}, x_{4} ; y\right)
$$

is skew in $x_{1}, x_{2}, x_{3}$ and if we let $x_{3}=z=x_{4}$ we obtain, using $\left(2.17^{\prime}\right)$,

$$
\begin{gathered}
Q\left(x_{1}, x_{2}, z, z ; y\right)-Q\left(x_{1}, z, x_{2}, z ; y\right)-Q\left(x_{1}, z, x_{2}, z ; y\right) \\
=-2 Q\left(x_{1}, x_{2} ; y, z\right) .
\end{gathered}
$$

Since $\operatorname{char} F \neq 2$, correcting $P$ by a multiple of this polynomial, we may assume that $P$ is alternating in $x_{1}, x_{2}, x_{3}, x_{4}$. As above $P$ is a linear combination of terms of the form $\{[],[],[]$,$\} . If the two y$ 's are in the end commuators, for example $\left\{\left[y, x_{1}\right]\left[x_{2}, x_{3}\right]\left[y, x_{4}\right]\right\}$, then exchanging the other two elements in these commutors yields the same term but with coefficient of opposite sign since $P$ is alternating in the $x$ 's. In the example $\left\{\left[y, x_{4}\right]\left[x_{2}, x_{3}\right]\left[y, x_{1}\right]\right\}=\left\{\left[y, x_{1}\right]\left[x_{2}, x_{3}\right]\left[y, x_{4}\right]\right\}$. Hence such terms have coefficient zero in $P$ and we are left with a multiple of

$$
\sum_{\sigma \in \mathscr{S}_{4}}(-1)^{\sigma}\left\{\left[y, x_{\sigma(1)}\right]\left[y, x_{\sigma(2)}\right]\left[x_{\sigma(3)}, x_{\sigma(4)}\right]\right\}=2 T_{6}\left(x_{1}, x_{2}, x_{3}, x_{4}, y ; y\right) \text {. }
$$

So $P$ is a consequence of $Q$ and $T_{6}$.

Finally we must consider multilinear identities of $H_{3}$ of degree 6 . Let $P\left(x_{1}, \ldots, x_{6}\right) \in I\left(H_{3}\right)$ be multilinear. The aim is to subtract from $P$ linear combinations of $Q$ 's and $T_{6}$ until $P$ is alternating. If we let two $x$ 's equal 
$y$ we get a linear combination of these $Q$ 's and a $G_{6}$ as was noted in the previous section. We may therefore assume that $P$ is skew in $x_{1}, x_{2}$. Then

$$
\begin{aligned}
& P\left(x_{1}, y, y, x_{4}, x_{5}, x_{6}\right)=\alpha_{1} Q\left(x_{1}, x_{4}, x_{5}, x_{6} ; y\right)+\alpha_{2} Q\left(x_{1}, x_{5}, x_{6}, x_{4} ; y\right) \\
& \quad+\alpha_{3} Q\left(x_{1}, x_{6}, x_{4}, x_{5} ; y\right)+\beta T_{6}\left(x_{1}, x_{4}, x_{5}, x_{6}, y ; y\right) .
\end{aligned}
$$

By (2.18), subtracting

$$
\begin{aligned}
& \alpha_{1} Q\left(x_{1}, x_{4}, x_{2}, x_{5}, x_{6}, x_{3}\right)+\alpha_{2} Q\left(x_{1}, x_{5}, x_{2}, x_{6}, x_{4}, x_{3}\right) \\
& \quad+\alpha_{3} Q\left(x_{1}, x_{6}, x_{2}, x_{4}, x_{5}, x_{3}\right)+\beta T_{6}\left(x_{1}, x_{4}, x_{5}, x_{6}, x_{2} ; x_{3}\right)
\end{aligned}
$$

yields an identity which is $x_{2}, x_{3}$ skew and still $x_{1}, x_{2}$ skew. We may therefore assume that $P$ is $x_{1}, x_{2}, x_{3}$ skew. So $P\left(x_{1}, x_{2}, y, y, x_{5}, x_{6}\right)$ is $x_{1}, x_{2}$ skew, which forces it to be of the form

$$
\begin{aligned}
& \alpha_{1} Q\left(x_{1}, x_{2}, x_{5}, x_{6} ; y\right)+\alpha_{2}\left(Q\left(x_{1}, x_{5}, x_{6}, x_{2} ; y\right)-Q\left(x_{1}, x_{6}, x_{2}, x_{5} ; y\right)\right) \\
& \quad+\beta T_{6}\left(x_{1}, x_{2}, x_{5}, x_{6}, y ; y\right) .
\end{aligned}
$$

\section{Subtracting}

$$
\begin{aligned}
& \alpha_{1} Q\left(x_{1}, x_{2}, x_{3}, x_{5}, x_{6}, x_{4}\right) \\
& \quad+\frac{\alpha_{2}}{2}\left(Q\left(x_{1}, x_{5}, x_{3}, x_{6}, x_{2}, x_{4}\right)-Q\left(x_{1}, x_{6}, x_{3}, x_{2}, x_{5}, x_{4}\right)\right. \\
& \quad-Q\left(x_{1}, x_{5}, x_{2}, x_{6}, x_{3}, x_{4}\right)+Q\left(x_{1}, x_{6}, x_{2}, x_{3}, x_{5}, x_{4}\right) \\
& \left.\quad-Q\left(x_{2}, x_{5}, x_{3}, x_{6}, x_{1}, x_{4}\right)+Q\left(x_{2}, x_{6}, x_{3}, x_{1}, x_{5}, x_{4}\right)\right) \\
& \quad+\beta T_{6}\left(x_{1}, x_{2}, x_{5}, x_{6}, x_{3}, x_{4}\right)
\end{aligned}
$$

yields an identity which is $x_{1}, x_{2}, x_{3}, x_{4}$ skew. Assume that $P$ is $x_{1}, x_{2}, x_{3}$, $x_{4}$ skew. We must have

$$
\begin{aligned}
P\left(x_{1}, x_{2}, x_{3}, y, y, x_{6}\right)= & \alpha\left(Q\left(x_{1}, x_{2}, x_{3}, x_{6} ; y\right)+Q\left(x_{1}, x_{3}, x_{6}, x_{2} ; y\right)\right. \\
& \left.-Q\left(x_{1}, x_{6}, x_{2}, x_{3} ; y\right)\right) \\
& +\beta T_{6}\left(x_{1}, x_{2}, x_{3}, x_{6}, y ; y\right) .
\end{aligned}
$$

Subtracting

$$
\begin{aligned}
& \alpha\left(Q\left(x_{1}, x_{2}, x_{4}, x_{3}, x_{6}, x_{5}\right)+Q\left(x_{1}, x_{3}, x_{4}, x_{6}, x_{2}, x_{5}\right)\right. \\
& \left.\quad-Q\left(x_{1}, x_{6}, x_{5}, x_{2}, x_{3}, x_{4}\right)-Q\left(x_{1}, x_{2}, x_{3}, x_{4}, x_{6}, x_{5}\right)\right) \\
& \quad+\beta T_{6}\left(x_{1}, x_{2}, x_{3}, x_{6}, x_{4} ; x_{5}\right)
\end{aligned}
$$

yields an identity which is $x_{1}, x_{2}, x_{3}, x_{4}, x_{5}$ skew.

Assume that $P\left(x_{1}, x_{2}, x_{3}, x_{4}, x_{5}, x_{6}\right) \in I\left(H_{3}\right)$ is multilinear and skew in $x_{1}, \ldots, x_{5}$. Then $P\left(x_{1}, x_{2}, x_{3}, x_{4}, y, y\right)$ is alternating in the $x$ 's. Using equation $\left(1.17^{\prime}\right)$ this implies that $P\left(x_{1}, x_{2}, x_{3}, x_{4}, y, y\right)$ is a multiple of $T_{6}\left(x_{1}, x_{2}, x_{3}, x_{4}, y ; y\right)$.

Proposition 11. If char $F \nmid 4$ ! then any homogeneous identity of $H_{4}(F)$ of degree 8 is a consequence of $T_{8}$.

Proof. By Proposition 9, $H_{4}$ has no identity of type $\left[3,1^{5}\right]$ or lower. We show next that it has no identity of types $\left[2^{4}\right],\left[2^{3}, 1^{2}\right]$ and $\left[2^{2}, 1^{4}\right]$. 
If $P\left(x_{1}, x_{2}, x_{3}, x_{4}\right) \in I\left(H_{4}\right)$ is of type [ $\left.2^{4}\right]$ then, by Osborn's theorem, it is symmetric in all four variables and, by Corollary $4, P$ is skew under the action of ${ }^{*} . P$ is a linear combination of products of four commutators. We will show that the coefficients of these terms must be zero. We say that two terms are equivalent if they are equivalent up to symmetry. Equivalent terms have the same coefficient. Any term is equivalent to a term which starts with $\left[x_{1}, x_{2}\right]$. If it also ends in $\left[x_{1}, x_{2}\right]$ then it must be $\left[x_{1}, x_{2}\right]\left[x_{3}, x_{4}\right]\left[x_{3}, x_{4}\right]\left[x_{1}, x_{2}\right]$ whose coefficient is zero by Lemma 5. If neither $x_{1}$ nor $x_{2}$ is present in the last commutator then the term ends in $\left[x_{3}, x_{4}\right]$. Since $\left[x_{1}, x_{2}\right]\left[x_{1}, x_{2}\right]$ has coefficient zero by Lemma 5 , up to equivalence we have $\left[x_{1}, x_{2}\right]\left[x_{3}, x_{4}\right]\left[x_{1}, x_{2}\right]\left[x_{3}, x_{4}\right]$ or $\left[x_{1}, x_{2}\right]\left[x_{1}, x_{3}\right]\left[x_{2}, x_{4}\right]\left[x_{3}, x_{4}\right]$. The first term starred is

$$
\left[x_{3}, x_{4}\right]\left[x_{1}, x_{2}\right]\left[x_{3}, x_{4}\right]\left[x_{1}, x_{2}\right]
$$

which is equivalent to $\left[x_{1}, x_{2}\right]\left[x_{3}, x_{4}\right]\left[x_{1}, x_{2}\right]\left[x_{3}, x_{4}\right]$. So its coefficient is zero. Similarly the coefficient of $\left[x_{1}, x_{2}\right]\left[x_{1}, x_{3}\right]\left[x_{2}, x_{4}\right]\left[x_{3}, x_{4}\right]$ is zero.

If $x_{1}$ or $x_{2}$ is present in the last commutator (but not both) the term is equivalent to $\left[x_{1}, x_{2}\right]\left[, x_{4}\right]\left[, x_{4}\right]\left[x_{1}, x_{3}\right]$. By Lemma 5 , the coefficient of $\left[x_{1}, x_{2}\right]\left[x_{2}, x_{4}\right]\left[x_{3}, x_{4}\right]\left[x_{1}, x_{3}\right]$ is minus the coefficient of

$$
\left[x_{1}, x_{2}\right]\left[x_{3}, x_{4}\right]\left[x_{2}, x_{4}\right]\left[x_{1}, x_{3}\right] \text {. }
$$

This term starred is $\left[x_{1}, x_{3}\right]\left[x_{2}, x_{4}\right]\left[x_{3}, x_{4}\right]\left[x_{1}, x_{2}\right]$ which is equivalent to $\left[x_{1}, x_{2}\right]\left[x_{3}, x_{4}\right]\left[x_{2}, x_{4}\right]\left[x_{1}, x_{3}\right]$. So its coefficient is zero and $P$ is identically zero. Thus $H_{4}$ has no identity of type $\left[2^{4}\right]$.

If $P\left(x_{1}, x_{2}, y_{1}, y_{2}, y_{3}\right) \in I\left(H_{4}\right)$ is of type $\left[2^{3}, 1^{2}\right]$, then by Osborn's theorem it is skew in the $x$ 's and symmetric in the $y$ 's. As usual we write $P$ as a linear combination of commutators and consider terms which could have different coefficients. If both $x$ 's are in the same commutator then, by Lemma 2.2 , it cannot be at the beginning or at the end since $H_{3}$ has no identity of type $\left[2^{3}\right]$. Starring if necessary, we may assume that $\left[x_{1}, x_{2}\right]$ is in second place. Such a term is equivalent to $\left[y_{1}, y_{2}\right]\left[x_{1}, x_{2}\right]\left[, y_{3}\right]\left[, y_{3}\right]$. By Lemma 5, we need only consider

$$
\left[y_{1}, y_{2}\right]\left[x_{1}, x_{2}\right]\left[y_{1}, y_{3}\right]\left[y_{2}, y_{3}\right] \text {. }
$$

Consider terms with the $x$ 's in distinct commutators. We may assume that $x_{1}$ comes before $x_{2}$. If the $x$ 's are in the first and second commutators, since by Lemma $5\left[x_{1}, y_{1}\right]\left[x_{2}, y_{1}\right]\left[y_{2}, y_{3}\right]\left[y_{2}, y_{3}\right]$ has coefficient zero, we need only consider

$$
\left[y_{1}, x_{1}\right]\left[y_{2}, x_{2}\right]\left[y_{1}, y_{3}\right]\left[y_{2}, y_{3}\right] \text {, }
$$

(exchanging $y_{1}$ and $y_{2}$ in the last two commutators results in a sign change by Lemma 5). Terms with the $x$ 's in the last two commutators are obtained by starring (2). For terms of the form $\left[, x_{1}\right][],\left[, x_{2}\right][$, ] we have

$$
\left[y_{1}, x_{1}\right]\left[y_{2}, y_{3}\right]\left[y_{1}, x_{2}\right]\left[y_{2}, y_{3}\right] \text {, }
$$


if the $x$ 's have the same mate, and

$$
\left[y_{1}, x_{1}\right]\left[y_{1}, y_{3}\right]\left[y_{2}, x_{2}\right]\left[y_{2}, y_{3}\right] \text {, }
$$

since the coefficient of $\left[y_{1}, x_{1}\right]\left[y_{2}, y_{3}\right]\left[y_{2}, x_{2}\right]\left[y_{1}, y_{3}\right]$ is minus the coefficient of $\left[y_{1}, x_{1}\right]\left[y_{2}, x_{2}\right]\left[y_{2}, y_{3}\right]\left[y_{1}, y_{3}\right]$ by Lemma 5 . As usual, starring will give us the coefficients of the terms of the form $[],\left[, x_{1}\right][],\left[, x_{2}\right]$. Moreover applying Lemma 5 to (4) yields the coefficient of $\left[y_{1}, y_{3}\right]\left[y_{1}, x_{1}\right]\left[y_{2}, x_{2}\right]\left[y_{2}, y_{3}\right]$. We need however

$$
\left[y_{2}, y_{3}\right]\left[y_{1}, x_{1}\right]\left[y_{1}, x_{2}\right]\left[y_{2}, y_{3}\right]
$$

and

$$
\left[y_{2}, y_{3}\right]\left[y_{1}, x_{1}\right]\left[y_{2}, x_{2}\right]\left[y_{1}, y_{3}\right] \text {. }
$$

Finally for terms of the form $\left[, x_{1}\right][],[],\left[, x_{2}\right]$, since

$$
\left[y_{1}, x_{1}\right]\left[y_{2}, y_{3}\right]\left[y_{2}, y_{3}\right]\left[y_{1}, x_{2}\right]
$$

has coefficient zero, we need only $\left[y_{1}, x_{1}\right]\left[y_{1}, y_{3}\right]\left[y_{2}, y_{3}\right]\left[y_{2}, x_{2}\right]$ (the middle $y_{1}, y_{2}$ can be interchanged) but this has minus the coefficient of (4). Let $\alpha_{1}, \ldots, \alpha_{6}$ be the coefficients in $P$ of the above six terms. Since $H_{4}$ has no identity of type $\left[3,2^{2}, 1\right]$, the polynomial $P\left(x_{1}, y_{1}, y_{1}, y_{2}, y_{3}\right)$ must be identically zero. Considering the coefficient of $\left[y_{1}, x_{1}\right]\left[y_{1}, y_{2}\right]\left[y_{1}, y_{3}\right]\left[y_{2}, y_{3}\right]$ yields

$$
\alpha_{4}=\alpha_{2} .
$$

The coefficient of $\left[y_{1}, y_{3}\right]\left[y_{2}, x_{1}\right]\left[y_{1}, y_{2}\right]\left[y_{1}, y_{3}\right]$ yields

$$
\alpha_{5}=-2 \alpha_{2} \text {. }
$$

The coefficient of $\left[y_{2}, x_{1}\right]\left[y_{1}, y_{3}\right]\left[y_{1}, y_{2}\right]\left[y_{1}, y_{3}\right]$ yields

$$
\alpha_{2}+\alpha_{3}+\alpha_{4}=0 \text {. }
$$

Using (7) we have

$$
\alpha_{3}=-2 \alpha_{2} \text {. }
$$

The coefficient of $\left[y_{1}, y_{2}\right]\left[y_{1}, x_{1}\right]\left[y_{1}, y_{3}\right]\left[y_{2}, y_{3}\right]$ yields

$$
\alpha_{1}+\alpha_{2}+\alpha_{4}=0 \text {, or } \alpha_{1}=-2 \alpha_{2} \text {. }
$$

The coefficient of $\left[y_{2}, y_{3}\right]\left[y_{1}, x_{1}\right]\left[y_{1}, y_{2}\right]\left[y_{1}, y_{3}\right]$ yields

$$
\alpha_{1}-\alpha_{2}+\alpha_{6}=0 \text {, or } \alpha_{6}=3 \alpha_{2} \text {. }
$$

Evaluating $P$ at $y_{1}=e[24], y_{2}=e[12], y_{3}=e[23], x_{1}=e[34]$ and $x_{2}=$ $e[44]$, and considering the coefficient of $e_{34}$ yields

$$
2 \alpha_{1}+2 \alpha_{2}=0
$$

which with (11) gives $\alpha_{2}=0$ and hence all $\alpha$ 's are zero. Therefore $H_{4}$ has no identity of type $\left[2^{3}, 1^{2}\right]$. 
If $P\left(x_{1}, x_{2}, x_{3}, x_{4}, y_{1}, y_{2}\right)$ is an identity of type $\left[2^{2}, 1^{4}\right]$, by Osborn's theorem, it is skew in the $x$ 's and symmetric in the $y$ 's. Write $P$ as

$$
R+\sum_{i<j}\left(\left[x_{i}, x_{j}\right] P_{i j}+P_{i j}^{\prime}\left[x_{i}, x_{j}\right]\right),
$$

where $R$ does not have any words starting or ending with two $x$ 's. Since $P^{*}=-P, P_{i j}^{\prime}=P_{i j}^{*}$. By Lemma 2.2, $P_{i j} \in I\left(H_{3}\right)$ and is of type $\left[2^{2}, 1^{2}\right]$ so by Corollary $4, P_{i j}^{*}=-P_{i j}$. In fact $P_{i j}$ must be a multiple $\alpha_{i j}$ of $Q_{i j}=$ $Q\left(x_{1}, \ldots, \hat{x}_{i}, \ldots, \hat{x}_{j}, \ldots, x_{4} ; y_{1}, y_{2}\right)$. Since $P$ is skew in the $x$ 's all $\alpha_{i j}$ 's are the same up to sign. Letting $\alpha_{12}=-\alpha$, we get

$$
P=R+\sum_{i<j}(-1)^{i+j} \alpha\left[\left[x_{i}, x_{j}\right], Q_{i j}\right] .
$$

$R$ is the alternating sum over all permutations of the $x_{j}$ 's of a linear combination of products of four commutators the first and last containing at least one $y$. To determine the coefficients, using the symmetry of $P$ in $y_{1}, y_{2}$, it suffices to consider terms starting in $\left[y_{1},\right]$. If it ends in $\left[y_{1}, x\right]$ then we need only

$$
\begin{aligned}
& {\left[y_{1}, y_{2}\right]\left[y_{2},\right][,]\left[y_{1},\right],} \\
& {\left[y_{1}, y_{2}\right][,]\left[y_{2},\right]\left[y_{1},\right],} \\
& {\left[y_{1},\right]\left[y_{2},\right]\left[y_{2},\right]\left[y_{1},\right] .}
\end{aligned}
$$

If it ends in $y_{2}$ then we must consider

$$
\begin{aligned}
& {\left[y_{1},\right]\left[y_{1},\right]\left[y_{2},\right]\left[y_{2},\right],} \\
& {\left[y_{1},\right]\left[y_{2},\right]\left[y_{1},\right]\left[y_{2},\right],} \\
& {\left[y_{1}, y_{2}\right][,][,]\left[y_{1}, y_{2}\right],}
\end{aligned}
$$

since $\left[y_{1}, y_{2}\right][],\left[y_{1},\right]\left[y_{2},\right]$ is gotten from permuting $y_{1}$ and $y_{2}$ in (15), and the coefficient of $\left[y_{1},\right]\left[y_{1}, y_{2}\right][],\left[y_{2},\right]$ is minus that of

$$
\left[y_{1}, y_{2}\right]\left[y_{1},\right][,]\left[y_{2},\right],
$$

that is, the coefficient of $\left[y_{2}, y_{1}\right]\left[y_{1},\right][],\left[y_{2},\right]$ which has the same coefficient as (14) by symmetry. The last four terms must have coefficient zero since $P^{*}=-P$. For example,

$$
\left[y_{1}, x_{1}\right]\left[y_{1}, x_{2}\right]\left[y_{2}, x_{3}\right]\left[y_{2}, x_{4}\right]^{*}=\left[y_{2}, x_{4}\right]\left[y_{2}, x_{3}\right]\left[y_{1}, x_{2}\right]\left[y_{1}, x_{1}\right]
$$

which has the same coefficient as $\left[y_{1}, x_{4}\right]\left[y_{1}, x_{3}\right]\left[y_{2}, x_{2}\right]\left[y_{2}, x_{1}\right]$ by symmetry; this term in turn has the same coefficient as $\left[y_{1}, x_{1}\right]\left[y_{1}, x_{2}\right]\left[y_{2}, x_{3}\right]\left[y_{2}, x_{4}\right]$ since it is obtained by the product of two transpositions. But it also has minus this coefficient since $P^{*}=-P$. Since char $F \neq 2$ this coefficient must be zero.

Let $\alpha_{1}, \alpha_{2}$ be the coefficients of (14) and (15) with the $x$ 's in order. Since $H_{4}$ has no identity of type $\left[3,2,1^{3}\right]$, the polynomial $P\left(x_{1}, x_{2}, x_{3}, y_{1} ; y_{1}, y_{2}\right)$ is identically zero. Considering the coefficient of $\left[y_{1}, x_{1}\right]\left[y_{1}, y_{2}\right]\left[y_{2}, x_{2}\right]\left[y_{1}, x_{3}\right]$, we get

$$
2 \alpha=0
$$


The coefficient of $\left[y_{1}, y_{2}\right]\left[y_{1}, x_{1}\right]\left[y_{2}, x_{2}\right]\left[y_{1}, x_{3}\right]$ yields

$$
\alpha_{2}=-\alpha \text {, }
$$

and the coefficient of $\left[y_{1}, y_{2}\right]\left[y_{1}, x_{1}\right]\left[y_{1}, x_{2}\right]\left[y_{2}, x_{3}\right]$,

$$
\alpha_{1}=\alpha_{2} .
$$

Therefore the coefficients are zero and $H_{4}$ has no identity of type $\left[2^{2}, 1^{4}\right]$.

If $P\left(x_{1}, \ldots, x_{6} ; y\right) \in I\left(H_{4}\right)$ is of type $\left[2,1^{6}\right]$ then either by Osborn's theorem or, if one prefers, since $H_{4}$ has no identity of type $\left[2^{2}, 1^{4}\right], P$ is skew in the $x$ 's. So $P$ is the alternating sum over all permutations of the $x$ 's of a linear combination of products of four commutators. Only four coefficients are needed, those of

$$
\begin{aligned}
& {[y,][,][y,][,],} \\
& {[y,][y,][,][,],} \\
& {[y,][,][,][y,],} \\
& {[,][y,][y,][,],}
\end{aligned}
$$

starring the first two gives the remaining two possibilities. By Lemma 2.2 and the form of $T_{6}\left(x_{1}, \ldots, x_{4}, y ; y\right)$, terms of the form (23) will not appear. Let $\alpha_{1}, \alpha_{2}, \alpha_{3}$ be the coefficients of (24), (25), and (26) in $P$, with the $x$ 's in order. Since $H_{4}$ has no identity of type $\left[3,1^{5}\right], P\left(x_{1}, x_{2}, x_{3}, x_{4}, x_{5}, y ; y\right)$ is identically zero. The coefficient of $\left[y, x_{1}\right]\left[y, x_{2}\right]\left[y, x_{3}\right]\left[x_{4}, x_{5}\right]$ yields

$$
\alpha_{1}+\alpha_{3}=0
$$

and that of $\left[y, x_{1}\right]\left[y, x_{2}\right]\left[x_{3}, x_{4}\right]\left[y, x_{5}\right]$ yields

$$
\alpha_{1}-\alpha_{2}=0 \text {. }
$$

Thus $P$ is a multiple of $G_{8}\left(x_{1}, x_{2}, x_{3}, x_{4}, x_{5}, x_{6} ; y\right)$.

Finally if $P\left(x_{1}, \ldots, x_{8}\right) \in I\left(H_{4}\right)$ is of type $\left[1^{8}\right]$, arguing as in the case of $H_{2}$ using the fact that identities of type $\left[2,1^{6}\right]$ come from $T_{8}$ we get $P$ alternating. This completes the proof of the proposition.

We have given this proof in great detail since it is important to know that $\mathrm{H}_{4}$ has no identity of type $\left[2^{2}, 1^{4}\right]$. The following proposition completes the proof of Theorem 1.2.

Proposition 12. If $|F| \geq 2 n$ and char $F+e(n)$ !, then for $n \geq 4$ any identity of $H_{n}(F)$ of degree $2 n$ is a consequence of $T_{2 n}$.

Proof. We prove the proposition by induction on $n$. It is true for $n=4$. By Proposition $9, H_{n}$ has no identity of type $\left[3,1^{2 n-3}\right]$.

Let $n>4$ and assume that the proposition is true for $n-1$. Let

$$
P\left(x_{1}, \ldots, x_{2 n-2} ; y\right)
$$


be an identity of $H_{n}$ of type $\left[2,1^{2 n-2}\right]$. For a fixed but arbitrary pair $i, j$, $1 \leq i<j \leq 2 n-2$,

$$
\begin{aligned}
P\left(x_{1}, \ldots, x_{2 n-2} ; y\right)= & \alpha_{i j} T_{2 n-2}\left(, \hat{x}_{i}, \ldots, \hat{x}_{j}, \ldots, y ; y\right)\left[x_{i}, x_{j}\right] \\
& +\beta_{i j}\left[x_{i}, x_{j}\right] T_{2 n-2}\left(\ldots, \hat{x}_{i}, \ldots, \hat{x}_{j}, \ldots, y ; y\right) \\
& + \text { terms which do not start or end in }\left[x_{i}, x_{j}\right] .
\end{aligned}
$$

Starring $P$ shows that $\beta_{i j}=-\alpha_{i j}$ and

$$
\begin{aligned}
P\left(x_{1}, \ldots, x_{2 n-2} ; y\right)= & \alpha_{i j}\left[T_{2 n-2}\left(, \hat{x}_{i}, \ldots, \hat{x}_{j}, \ldots, y ; y\right),\left[x_{i}, x_{j}\right]\right] \\
& + \text { terms which do not start or end in }\left[x_{i}, x_{j}\right] .
\end{aligned}
$$

Doing this for different choices of $i, j$ and comparing the coefficients of common terms shows that the $\alpha$ 's are the same up to sign. In this way we get that $P$ is a multiple of $T_{2 n}\left(x_{1}, \ldots, x_{2 n-2}, y ; y\right)$ except possibly for terms starting and ending in $[y$,$] . For \sigma \in \mathscr{S}_{2 n-2}$, letting $x_{\sigma(2)}=y$ and using the fact that $H_{n}$ has no identity of type $\left[3,1^{2 n-3}\right]$, the coefficient of

$$
\left[y, x_{\sigma(1)}\right]\left[y, x_{\sigma(3)}\right]\left[x_{\sigma(4)}, x_{\sigma(5)}\right] \cdots\left[y, x_{\sigma(2 n-2)}\right]
$$

will tell us that no terms of the form $\left[y, x_{\sigma(1)}\right]\left[x_{\sigma(2)}, x_{\sigma(3)}\right] \cdots\left[y, x_{\sigma(2 n-2)}\right]$ have nonzero coefficient in $P$ if $n$ is odd and that they have the right coefficient for $P$ to be a multiple of $T_{2 n}\left(x_{1}, \ldots, x_{2 n-2}, y ; y\right)$ if $n$ is even.

If $P\left(x_{1}, \ldots, x_{2 n}\right)$ is a multilinear identity of $H_{n}$ then using the fact that any identity of type $\left[2,1^{2 n-2}\right]$ comes from $T_{2 n}$ we can modify $P$ by subtracting multiples of $T_{2 n}$. We get $P$ alternating and thus a multiple of $S_{2 n}$. This completes the proof of the proposition and of Theorem 1.2.

Remarks. (1) It should be possible to weaken the assumption on the characteristic of $F$, by considering the identities which are consequences of $T_{2 n}$ and $S_{2 n}$ when char $F \mid e(n)$.

(2) While $I\left(H_{n}\right)$ is not a $T$-ideal it is stable under permutation of the variables and substitution of Jordan polynomials.

(3) Amitsur has shown that $S_{2 n}$ does not generate $I\left(M_{n}(F)\right)$. Using Lemma 2.4, his argument [7, Proposition 2.4.23] will show that $T_{2 n}$ does not generate $I\left(H_{n}(F)\right)$.

\section{REFERENCES}

1. A. S. Amitsur and J. Levitzki, Minimal identities for algebras, Proc. Amer. Math. Soc. 1 (1950), 449-463.

2. Claude Berge, Graphes et hypergraphes, Dunod, Paris, 1970.

3. N. Jacobson, Structure and representations of Jordan algebras, Amer. Math. Soc. Colloq. Publ., vol. 39, Amer. Math. Soc., Providence, R.I., 1968.

4. J. Marshall Osborn, Identities of non-associative algebras, Canad. J. Math. 17 (1965), 78-92.

5. Y. P. Razmyslov, Finite basing of the identities of a matrix algebra of second order over a field of characteristic 0, Algebra i Logika 12 (1973), 47-63. 
6. - Trace identities of full matrix algebras over a field of characteristic zero, Math. USSRIzv. 8 (1974), 727-760.

7. L. H. Rowen, Polynomial identities in ring theory, Academic Press, 1980.

8. A simple proof of Kostant's theorem and an analogue for the symplectic involution, Contemp. Math., vol. 13, Amer. Math. Soc., Providence, R.I., 1982, pp. 207-215.

9. A. M. Slin'ko, Special varieties of Jordan algebras, Mat. Zametki 26 (1979), 337-344.

10. B. D. Smith, A standard Jordan polynomial, Comm. Algebra 5 (1977), 207-218.

11. Richard G. Swan, An application of graph theory to algebra, Proc. Amer. Math. Soc. 14 (1963), 367-373; Correction, Proc. Amer. Math. Soc. 21 (1969), 379-380.

Department of Mathematics, University of Ottawa, Ottawa, Ontario, Canada Kin $6 \mathrm{~N} 5$ 